\title{
Capacity Optimization and Configuration for Household Wind/PV/ Storage Hybrid Power Generation System
}

\author{
Min Wang \\ Mechanical School, Southeast University, Nanjing 210000
}

Abstract: For capacity matching and design calculation on off-grid Hybrid Renewable Energy System, commercial software like Homer developed by the National Renewable Energy Laboratory is commonly used at present. This paper takes the load demand of household users as the research object, and uses the three cities with different potentials of wind and solar resources as the of the Wind/PV/Storage hybrid power generation systems simulation installation site. The Homer optimizes the system capacity configuration, and the goal is to find the minimum investment cost while meeting the users electricity demand. The results show that the potential of the wind and solar resources will affect the levelized cost of energy of the installed system. The levelized cost of energy will be smaller in the place where the wind and solar resources are abundant. At the same time, the unit cost of the wind turbine is higher than that of the Photovoltaic system, so the cost of wind turbines effect is more obvious for the levelized cost of energy of the overall system. Finally, the paper also analyzes the sensitivity of the technical parameters of wind turbines and loss of power supply probability.

Keywords: Energy and Power Engineering; Hybrid Renewable Energy System; Capacity Optimization and Configuration; Levelized Cost of Energy; Loss of Power Supply Probability

\section{Introduction}

Hybrid Renewable Energy System (HRES) is a combination of renewable energy, traditional energy and energy storage equipment. When HERS is applied in practice, in order to obtain the lowest investment and operating costs and meet the technology requirements and carbon emission limits should be optimized to determine the optimal size of the hybrid renewable energy system. At present, one of the most powerful tools to solve this problem is homer (hybrid optimization model for electric renewable), developed by the U.S. national renewable energy laboratory. this software has been widely used by many researchers around the world.

$\mathrm{Ma} \mathrm{Tao^{[2] }}$ et al. conducted a detailed feasibility study on an independent hybrid solar and wind energy system with battery energy storage. and technical and economic evaluation. The study recorded the solar radiation and wind data of the island in 2009 and used HOMER software for simulation and technical and economic evaluation. In terms of net system cost (NPC) and energy cost (COE), thousands of case studies are simulated to achieve the best system configuration. In addition, the author also studied the influence of solar panel size, wind turbine size and battery pack capacity on system reliability and economic performance. Finally, sensitivity analysis is carried out on its load consumption and renewable energy to evaluate the robustness of economic analysis and determine which variables have the greatest impact on the results. The results prove that the implementation of wind and solar energy reserve power supply. Technical and economic feasibility of 50 system to supply power to the island.

Ramli ${ }^{[3]}$ and others have studied the application of wind-solar hybrid power supply system in the west coast of Arab, where the annual average solar irradiance and wind speed are $5.95 \mathrm{kWh} / \mathrm{m}^{2} *$ Day and $3.53 \mathrm{~m} / \mathrm{s}$, respectively.

This is an open-access article distributed under the terms of the Creative Commons Attribution Non-Commercial License (http://creativecommons. org/licenses/by-nc/4.0/), which permits unrestricted non-commercial use, distribution, and reproduction in any medium, provided the original work is properly cited. 
Researchers have analyzed the technical feasibility and economy of hybrid energy system using MATLAB and Homer. Homer can model the hybrid renewable energy system with a power ratio ranging from $0.626 \mathrm{~kW}$ to $2213000 \mathrm{~kW}$.

\section{Resources and loads}

\subsection{Scenery resources potential}

Due to the difference of climate in different installation sites, especially the difference of wind speed and solar irradiance, the configuration and selection of wind, solar and energy storage systems studied in this paper will be different. Therefore, in the simulation, this paper selected several representative cities: Nanjing, Kinmen and Urumqi are the simulated installation locations. The data of solar irradiance, wind speed and temperature in this study are taken from National Aeronautics and Space Administration (NASA) and Space Administration, or NASA, The Langley Research Center (LaRC) Global Resource Forecast (The Forecast of World Wide Energy Resource project, which is funded by NASA's Earth Science/Applied Science Program, aims to pass the World Climate Research Program, the Global Energy and POWER Cycle Experiment, and the ground radiation budget, etc. Research to provide users with data services on climate and other related aspects ${ }^{[4]}$.

\subsection{Load demand}

In the simulation, the average daily load demand of users is $11.26 \mathrm{kwh}$ and the peak load power demand is $2.09 \mathrm{kw}$. Users have an obvious peak of 80 every day during the period of 18:00-21:00 at night, while others have a small range in the morning and noon. Demand for electricity has increased, which is basically in line with the electricity usage habits of ordinary households.

The simulation period of the simulation system in this study is one year, and the time step is 1 hour, i.e. each optimization is a whole year. The 8760 hours are based on the results of many simulations of energy balance. Energy balance is the core constraint condition in the optimization process, i.e. to ensure that the electricity demand of users at a certain moment is equal to the sum of the power generation amount of renewable energy and the power supply amount of energy storage.

\section{System configuration}

Homer provides users with a rich variety of components, and users can make choices according to their own system design requirements during the modeling process. In this paper, the wind turbine with rated power of $650 \mathrm{~W}$, the solar panel assembly with maximum power of $145 \mathrm{~W}$ and the battery unit with capacity of 2 kwh are selected for physical modeling. The model built in Homer is shown in Figure 1.

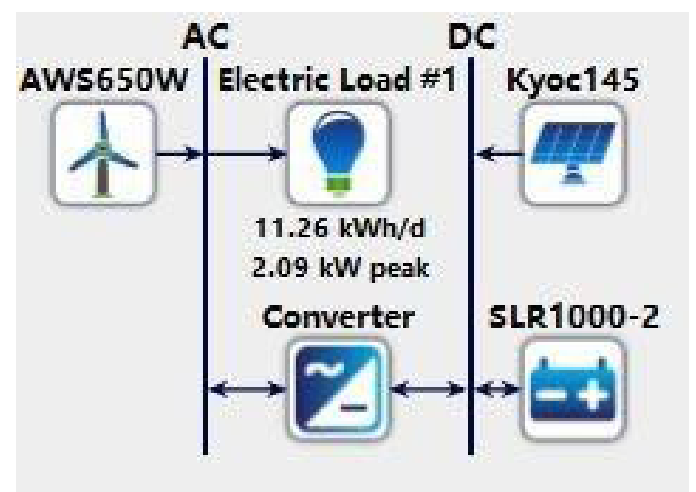

Figure 1. Schematic diagram of hybrid renewable energy system model.

In the optimization simulation stage, Homer will traverse all configuration combinations of the system according to the input data to carry out simulation; After excluding technically infeasible schemes, the net present value of the system for calculating feasible schemes is the discounted value of all investment, installation, operation and maintenance costs in the whole life cycle, i.e. the value of NPC, and the feasible schemes are sorted from low to high according to the size of NPC, and also according to different types system configuration scheme to sort; The lowest feasible plan for NPC is the optimal plan $^{[5]}$. The objective of optimization is to obtain the smallest configuration scheme of NPC, and then output 
the number of components of various types of optimal configuration schemes, NPC and economic analysis report, etc. Constraint conditions include energy supply and demand balance constraint, battery charge and discharge constraint and technical parameter constraint of each component. The maximum number of iterations in this study is set to 10000 .

\section{Result analysis}

The normalized energy cost (COE) in the results is defined as the useful electric energy generated by the energy system, and the average cost per kilowatt-hour is divided by the total annual electricity demand. The loss of power supply probability (LPSP) is defined as the ratio of the value of the annual load loss to the total load demand ${ }^{[6]}$.

Kinmen has the lowest COE because of its advantages in wind and light resources. Comparing the simulated installation sites of Nanjing and Urumqi, Nanjings wind energy potential is better than Urumqis, but Urumqis solar energy potential is better than Nanjings. Further analysis revealed that: The annual working hours of wind turbines in Nanjing are 7,698 hours, which is nearly 2,000 hours more than that in Urumqi. This is the main reason for the gap between the two wind turbines COE. The COE of solar panels in the two places is similar, which should be because the unit cost of wind turbine is higher than that of solar panels, so the cost of wind turbine in the system has a more significant impact on the total cost than that of solar panels.

It can be seen that different COE can be obtained when different wind turbines are used to build the 115 model, which indicates that the wind turbine parameters have an impact on the system COE. Although the two are not linearly related, the wind turbine with rated power of $0.65 \mathrm{kw}$ has the lowest system COE in this case.

When the wind-solar storage power supply system is used in the court, the wind turbine with smaller rated power should be selected as far as possible decrease gradually. Because when the allowable load power failure rate increases, it means that the design requirements can be met as long as the smaller load demand is met, so the corresponding investment in the system will be reduced, and the system COE will naturally be reduced. In actual operation, the user's family can access the power grid, so the unsatisfied load demand of renewable energy can be supplemented by the power grid. When designing the system, if the user's investment budget is limited, then a certain load power failure rate can be set, thus reducing the investment and obtaining the user's acceptance.

\section{Conclusion}

In this paper, Homer is used to analyze the technology and economy of the wind, light and storage power supply system for home use, and the optimal size scheme for each component configuration of the system is obtained, which provides reasonable suggestions for home users to apply the wind, light and storage system. Using Homer modeling and simulation optimization, it is necessary to provide relevant climate data such as wind speed, solar irradiance and temperature of the system simulation installation site. In order to explore the impact of these uncertain climate parameters on the output results, this paper selects three locations with different potential of wind and light resources, and compares the output results of the optimal allocation schemes in the three locations, and draws a conclusion that the potential of wind and light resources will affect the normalized energy cost COE of the installation system. Generally, the more abundant the wind and light resources are, the smaller the wind and light storage system COE installed in the locations will be. At the same time, because the unit cost of wind turbine is higher than that of solar panel, the cost of wind turbine has a more significant impact on the COE of the overall system. Through sensitivity analysis, it is suggested that wind turbines with smaller rated power should be selected when designing wind-solar storage power supply systems for families. The investment can be reduced by setting the load power failure rate to obtain an optimization scheme acceptable to users.

\section{References}

1. Bahramara S, Moghaddam M P, Haghifam M R. Optimal planning of hybrid renewable energy systems using HOMER: A review[J]. Renewable and Sustainable Energy Reviews, 2016, 62: 609-620.

2. Ma T, Yang H, Lu L. A feasibility study of a stand-alone hybrid solar-wind-battery system for a remote island[J]. Applied Energy, 2014, 121: 149-158.

3. Ramli M A M, Hiendro A, Al-Turki Y A. Techno-economic energy analysis of wind/solar hybrid system: Case study for western coastal area of Saudi Arabia[J]. Renewable energy, 2016, 91: 374-385. 
4. Can D A, Güler Onder. Techno-Economic Analysis of Off-Grid PV/Wind/Fuel Cell Hybrid System Combinations with a Comparison of Regularly and Seasonally Occupied Households[J]. Sustainable Cities and Society, 2018,42:107-126.

5. Khan F A, Pal N, Saeed S H. Review of solar photovoltaic and wind hybrid energy systems for sizing strategies optimization techniques and cost analysis methodologies[J]. Renewable and Sustainable Energy Reviews, 2018, 92: 937-947.

6. Tian Hongxiang, Liu Dawei, Zhu Lianjun, et al. Optimization of battery capacity in wind-solar energy storage system [J]. Power Technology, 2018, 42 (3): 463-466. 